\section{GW23-e1504 THE EFFECT OF DEPRESSION ON PREMATURE VENTRICULAR CONTRACTIONS (PVCS) IN CORONARY HEART DISEASE (CHD) PATIENTS UNDERGOING PERCUTANEOUS CORONARY INTERVENTION}

doi:10.1136/heartjnl-2012-302920e.4

Wu Keng, Wu Keng. The Affiliated Hospital of Guang Dong Medical College

Objectives To determine whether Depression increases the incidence rate of premature ventricular contractions (PVCs) in CHD patients undergoing PCI.

Methods From November 1, 2010 and ended on October 30, 2011.160 CHD patients from single center were assigned to either the depression or the non-depression group according a validated Chinese version of Beck Depression Inventory (BDI). PVCs was required Lown Classification. The primary end point was the occurrence of SCDs at 6 months, including ICD, Cardiopulmonary Resuscitation (CPR). Secondary end point was antiarrhythmic drug (AAD) administration.

Results At 3 months, PVCs (Lown I-IVA) and AAD administration rates were significantly different between the depression $(33.7 \%$ and $43.5 \%)$ and the non-depression groups (15\% and $17.5 \%, \mathrm{p}<0.05)$. At 6-month, additional PVCs (Lown IVB-V) in the depression group $(7.5 \%)$ in occurring significantly more than in the non-depression group $(2.5 \%, \mathrm{p}<0.05)$.there were nonsignificant differences in SCDs, CPR and ICD between the depression (5\%, 2.5\%and 3.8\%) and nondepression groups $(2.5 \%, 2.5 \%$ and $4 \%, p>0.05)$.

Conclusions depression was associated with a significant increase of PVCs and $\mathrm{AAD}$ administration in this $\mathrm{CHD}$ patient population underwenting PCI. However, there was no significant difference in SCDs, CPR and ICD between the depression and the non-depression groups. 